Check for updates

Cite this: RSC Adv., 2017, 7, 18838

Received 29th December 2016 Accepted 14th March 2017

DOI: $10.1039 / c 6 r a 28803 k$

rsc.li/rsc-advances

\section{Durable antibacterial finishing of cotton fabric based on thiol-epoxy click chemistry}

\begin{abstract}
Dan Yu, (D)*ab Lijin Xu, ${ }^{a}$ Yi Hu, ${ }^{a}$ Yani $\mathrm{Li}^{\mathrm{a}}$ and Wei Wang ${ }^{\mathrm{a}}$
This research proposes a method based on thiol-epoxy click chemistry to achieve durable antibacterial properties on cotton fabrics. The cotton fabric was first modified with 3-mercaptopropyltriethoxysilane $(\mathrm{KH}-580)$ to introduce thiol groups. Then, cotton fabric was treated with a quaternary ammonium salt by thiol-epoxy click chemistry. The surface morphology of the treated fabrics and the reaction mechanism were confirmed by FTIR, EDS, and SEM. The antibacterial activity, dyeing performance, and mechanical properties of the treated cotton fabrics were also assessed. The E. coli antibacterial rate was about $99.44 \%$ and the S. aureus antibacterial rate was about $95.93 \%$, with only a slight decrease after 30 cycles of washing, to $93.89 \%$ and $88.62 \%$, respectively. The results demonstrated that this treatment effectively imparted the cotton fabric with durable antibacterial properties due to the chemical bonding formed between the quaternary ammonium salt and the substrate.
\end{abstract}

\section{Introduction}

Textiles with enhanced resistance against microorganisms (antibacterial, antimicrobial, antifungal textiles, etc.) are becoming increasingly desirable for textile manufacturers. In general, antibacterial properties can be imparted to the textile materials by chemically or physically incorporating functional agents to the fibers of the fabrics. ${ }^{1}$ Cotton is one of the excellent natural materials, but it is easy for bacteria to grow on it. ${ }^{2,3} \mathrm{As}$ a hydrophilic fabric textile, cotton easily provides suitable conditions, such as humidity, adequate temperature, and nutrition, for the growth of microorganisms. To a certain extent, this can generate an unpleasant odor, stains, and discoloration in the fabric. Therefore, it becomes necessary to apply an antibacterial finishing on cotton fabrics. Furthermore, the ideal antibacterial finishing should be non-toxic, washable, environmentally friendly, and durable.

The general methods for antibacterial finishing on cotton involve the impregnation of limonene microcapsules, ${ }^{4}$ chitosan/ TPP self-assembled nanolayers, ${ }^{5}$ PAMAM $/ \mathrm{Ag}^{+},{ }^{6}$ quaternary ammonium salts, ${ }^{7,8}$ and reactive siloxane sulfopropylbetaine. ${ }^{9}$ The impregnation with limonene microcapsules provides fragrance, but the antibacterial effect is not durable. On the other hand, the chitosan/TPP self-assembled nanolayers can improve the stability of nanoparticles in a disperse system. Moreover, large numbers of reactive groups are introduced on the surface of the nanolayers ${ }^{10}$ to improve the antibacterial

${ }^{a}$ College of Chemistry, Chemical Engineering and Biotechnology, Donghua University, 2999 North Renmin Road, Songjiang District, Shanghai 201620, China. E-mail: yudan@dhu.edu.cn; Fax: 86-21-67792608; Tel: 86-21-67792456

${ }^{b}$ Saintyear Holding Group Co., Ltd, China activity. However, nanolayers suffer from the limitation of being costly and are therefore unfeasible for large-scale use. Although antibacterial finishing with PAMAM/ $/ \mathrm{Ag}^{+}$possesses good properties and is resistant to washing, the silver excess will degrade the whiteness and mechanical properties of the textile. Among numerous available varieties of antibacterial agents, species containing a quaternary ammonium cation (QAC) have been widely used for decades to disinfect environmental surfaces, for instance, in clinical and industrial settings. This particular interest originates from inherent advantages including a broad activity spectrum against Gram-negative and Gram-positive bacteria, yeasts, or moulds, as well as effectiveness over a wide pH range. ${ }^{11-14}$ However, QAC-based species still present some problems such as poor adhesion and durability, particularly during padding and curing. In previous studies, activated carbon was functionalized with two types of quaternary ammonium groups to achieve antibacterial properties. ${ }^{15}$ The deposition of QAC-derived compounds on various types of supports can be achieved through physical techniques. However, the limitation of these methods is that the resulting QAC-treated textiles have poor durability, especially after various cycles of water washing. Antibacterial cotton textiles finished with reactive siloxane sulfopropylbetaine possess a durable antibacterial activity due to their covalent bonds, are environmentally friendly, and have less hydrophilicity and better breaking strength. Therefore, chemical modification is a good strategy to improve the durability and washing performance of textiles.

Recently, click chemistry ${ }^{16-19}$ has emerged as a powerful synthetic tool to access a number of drugs, polymers, and functional materials, and to conduct surface modifications, because of its high efficiency, versatility, and simplicity. Click chemistry is also widely used for textile functional finishes, such 

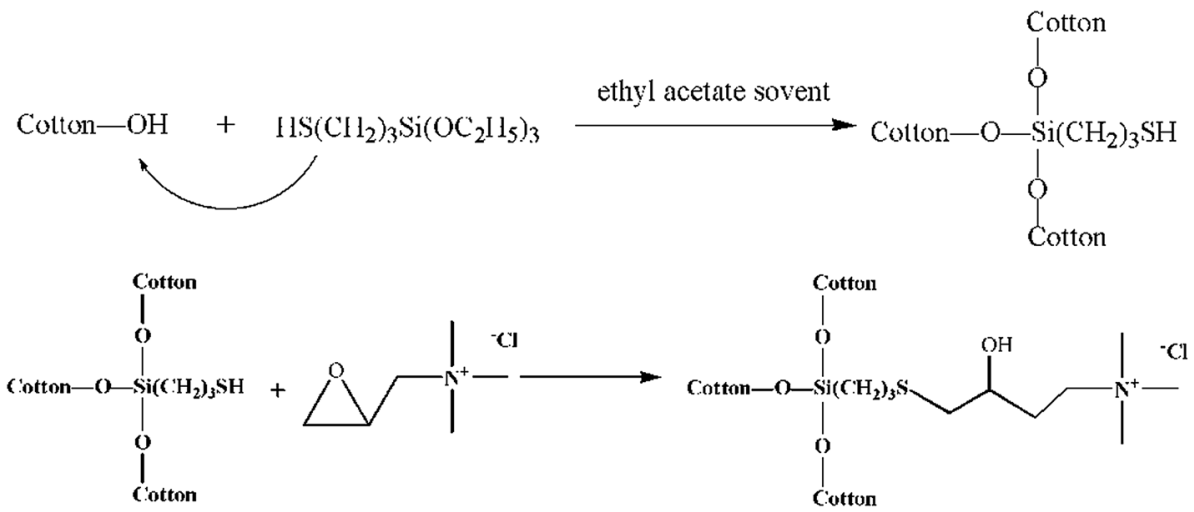

Scheme 1 Reaction scheme for the modification of the cotton fabric: (1) pretreatment with 3-mercaptopropyltriethoxysilane (KH-580) and (2) reaction of GTAC with the thiol groups in the cotton fabric by thiol-epoxy click chemistry.

as antibacterial, antistatic, flame retardant, etc., and has excellent application prospects. W. Tian, Y. Hu, et al. have successfully synthesized a gemini quaternary ammonium salt and applied it to cotton fabrics to achieve antibacterial properties. ${ }^{20}$ Additionally, modifications of the surface resistivity and control of moisture properties in keratin fibers can be carried out through thiol-ene click reactions. ${ }^{21}$ Furthermore, Ma et al. have discovered the click synthesis of quaternized poly functionalized graphene oxide, which also can improve the antibacterial and antifouling properties of textiles. ${ }^{22}$ Thus, all the abovementioned studies demonstrates the feasibility of applying click chemistry to the functional finishing of textiles.

In this study, the thiol-reactive groups of cotton were introduced through a condensation reaction between 3-mercaptopropyltriethoxysilane (KH-580) and the fiber. The grafting occurs by the reaction of the ethoxy group on one end of KH-580 and the hydroxyl groups of the fiber surface. Then, the cotton fabric with thiol groups and quaternary ammonium salts with epoxy structural chemicals (GTAC) form a strong chemical bond. Thus, this study reports a novel method to modify a cotton fabric to achieve antibacterial properties by thiol-epoxy click chemistry. The related reaction scheme is presented in Scheme 1. The final cotton fibers are expected to exhibit a permanent antibacterial property and good dyeing performance.

\section{Experimental}

\subsection{Materials}

A plain cotton weaved fabric of $230 \mathrm{~g} \mathrm{~m}^{-2}$ (Saintyear Holding Group Co., Ltd, China) was used in this study. 3-Mercaptopropyltriethoxysilane (KH-580) was purchased from Sigma Aldrich (USA). Glycidyl trimethyl ammonium chloride (GTAC) was obtained from Adamas. All other chemicals were of analytical grade and used without further purification (Sinopharm, China).

\subsection{Treatments}

Coarsening of cotton fabric was carried out by treating the fabric in a sodium hydroxide and levelling agent $\mathrm{O}$ (alkylphenol ethoxylates) solution in water at $70{ }^{\circ} \mathrm{C}$ for $20 \mathrm{~min}$. Then, 3mercaptopropyltriethoxysilane ( $\mathrm{KH}-580)$ was dissolved in ethyl acetate to a concentration of $5 \%$. Cotton fabrics were treated in the KH-580 solution at room temperature for $2 \mathrm{~h}$ at the liquor ratio of $1: 50$ under stirring. Subsequently, the KH-580-pretreated cotton fabrics were immersed in a water : ethanol $1: 1$ v/v) $5 \%$ GTAC solution, at the liquor ratio of $50: 1 .{ }^{23,24} \mathrm{The} \mathrm{pH}$ value was adjusted to 8.5 with $\mathrm{Na}_{2} \mathrm{CO}_{3} / \mathrm{NaHCO}_{3}$ buffer, which played a catalytic role. The reaction was completed after $8 \mathrm{~h}$ at $40{ }^{\circ} \mathrm{C}$ under gentle stirring. Finally, the cotton samples were rinsed with deionized water and dried at $60{ }^{\circ} \mathrm{C}$.

\subsection{Characterization}

2.3.1 Fourier transform infrared spectroscopy analysis. The samples were cut into pieces and analyzed by FT-IR with a Spectrometer Varian 640-IR (Varian, USA) at the resolution of 1 $\mathrm{cm}^{-1}$ by the potassium bromide pellet method. The number of scans was 32 .

2.3.2 Energy dispersive spectrometer. The chemical composition of the fiber surfaces was investigated by energy dispersive spectroscopy (EDS, IE 3000, U.K.).

2.3.3 Electronic scanning microscopy. The untreated and treated fabric samples were sputtered with a gold coating and then analyzed via a Desktop Scanning Electron Microscope TM1000 (Hitachi, Japan). The SEM images were obtained under vacuum at a $5000 \times$ magnification and at the accelerating voltage of $10 \mathrm{kV}$.

2.3.4 Antibacterial activity test. The antibacterial activity of the treated and untreated cotton fabrics was tested against $E$. coli and S. aureus according to the AATCC100-2004 standard. A $0.75 \mathrm{~g}$ sample was cut into $5 \mathrm{~mm} \times 5 \mathrm{~mm}$ squares to obtain the test samples, and three test samples were tested in parallel for each fabric. All the samples, glassware, broth, and medium used in the experiments were sterilized at $121{ }^{\circ} \mathrm{C}$ for $15 \mathrm{~min}$. $E$. coli was cultured in $100 \mathrm{~mL}$ of broth containing $3 \mathrm{~g} \mathrm{~L}^{-1}$ tryptone and $5 \mathrm{~g} \mathrm{~L}^{-1}$ beef extract at $37^{\circ} \mathrm{C}$ for $18-24 \mathrm{~h}$. The $\mathrm{pH}$ value was adjusted to 6.8-7.2 using a sodium carbonate solution. The medium contained an extra $20 \mathrm{~g} \mathrm{~L}^{-1}$ agar compared to broth. All the samples were cultivated in a $70 \mathrm{~mL}$ PBS buffer solution containing $5 \mathrm{~mL}$ of broth previously prepared at $37^{\circ} \mathrm{C}$ for $24-$ 

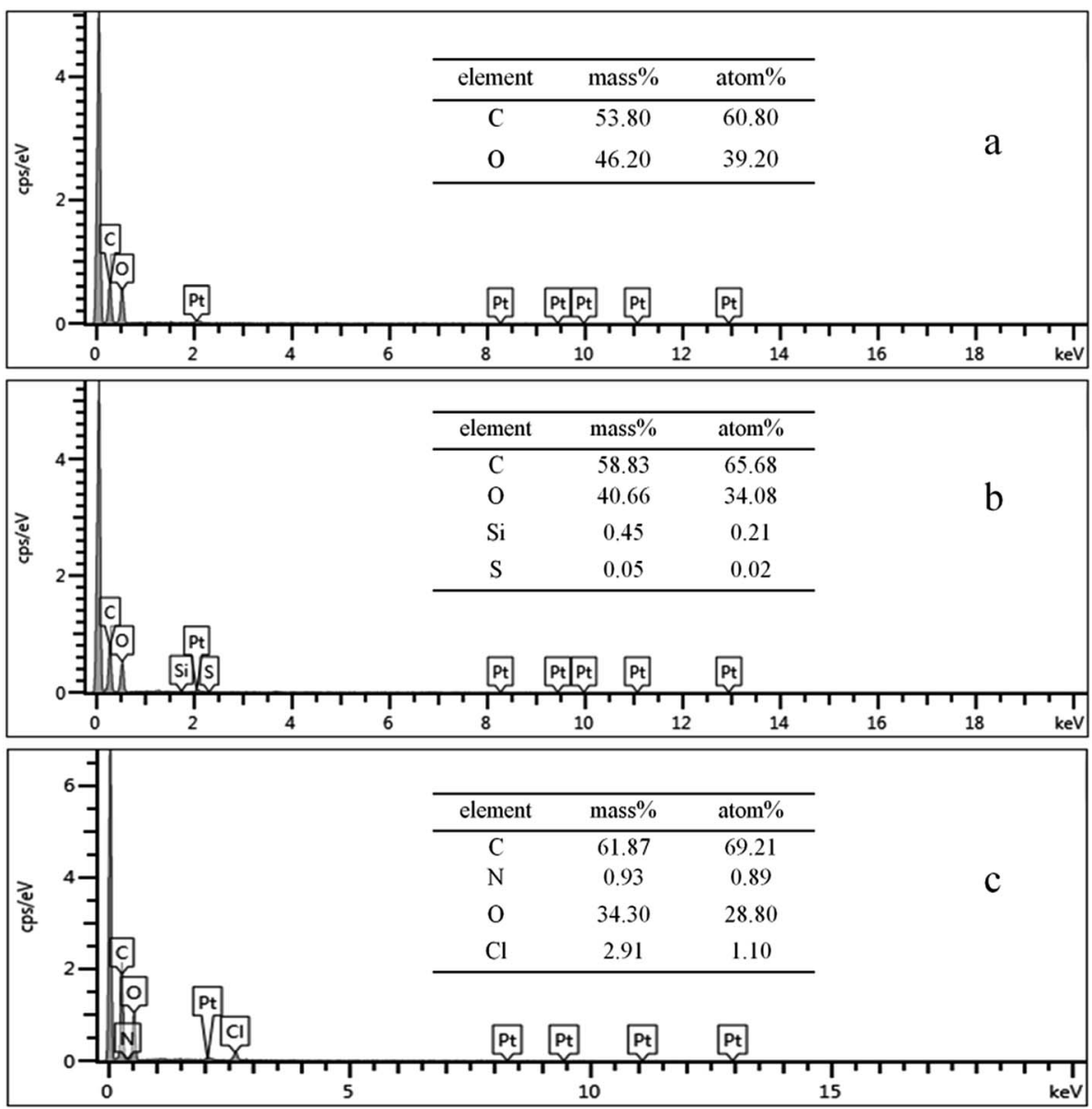

Fig. 1 EDS spectra of (a) untreated, (b) KH-580-pretreated, and (c) KH-580-GTAC-treated samples.

$48 \mathrm{~h}$. Then, a series of broths were used for the flat coating operation. The number of colonies was counted after 24-48 h. The reduction percentage of bacteria by the specimens was calculated by the following formula: ${ }^{25}$

$$
Y=((W-Q) / W) \times 100 \%
$$

where $Y$ is the antibacterial activity, $Q$ is the number of $\mathrm{CFU}$ recovered from the antibacterial samples or untreated samples incubated over $24 \mathrm{~h}$, and $W$ is the number of CFU recovered from the blank samples.

2.3.5 Staining test. The dyeing performance of cotton fabrics was tested via a Datacolor spectraflash SF600 Computer color-Matching System. The treated and untreated cotton samples were dyed in four baths using a reactive dye (Reactive Brilliant Red 3B, milling, C.I. number: Reactive Red 2, CAS: 17804-49-8, Hangzhou Anlongda Chemical Co., Ltd., China) to assess the dyeability changes of the fabrics before and after the treatments. The dye baths were set at $60^{\circ} \mathrm{C}$ with $20 \mathrm{~g} \mathrm{~L}^{-1} \mathrm{NaCl}$, $15 \mathrm{~g} \mathrm{~L}^{-1} \mathrm{Na}_{2} \mathrm{CO}_{3}$, and 4\% (o.w.f) Reactive Brilliant Red 3B (o.w.f stands for on weight of fabric, which is the reagent weight/fabric weight ratio, e.g., for each one hundred kilograms of fabric, the weight of other reagents $=100 \times x \%$ (o.w.f) $\mathrm{kg}$ ). After dyeing, the temperature was slowly increased to $90{ }^{\circ} \mathrm{C}$ and maintained at $90{ }^{\circ} \mathrm{C}$ for $30 \mathrm{~min}$. At the end of fixation, the fabric samples were thoroughly rinsed in warm and cold water and oven-dried at $50{ }^{\circ} \mathrm{C}$.

2.3.6 Strength test. The single fiber strength of the treated and untreated fabric samples was tested by Materials Testing Machine H5K-S (Tinius Olsen, USA). Before tests, cotton samples were placed in a room at constant temperature and

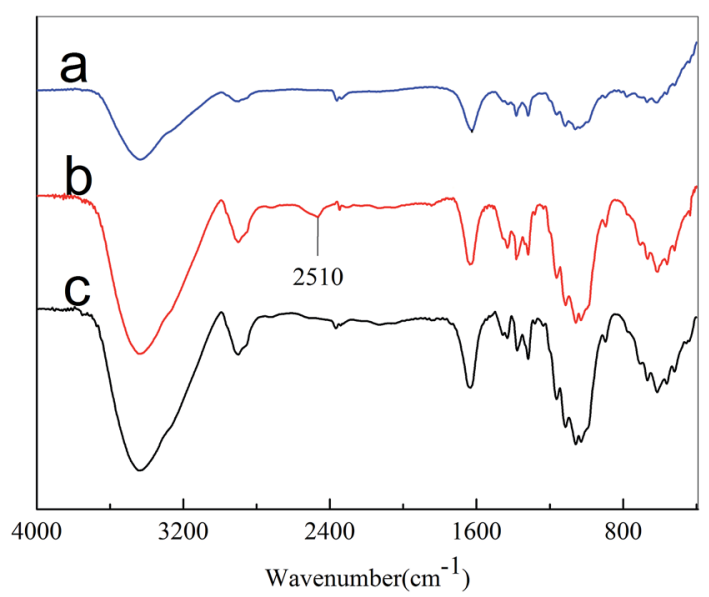

Fig. 2 IR spectra of the cotton samples: (a) untreated, (b) $\mathrm{KH}-580$ pretreated, and (c) $\mathrm{KH}-580-\mathrm{GTAC}$-treated samples. 

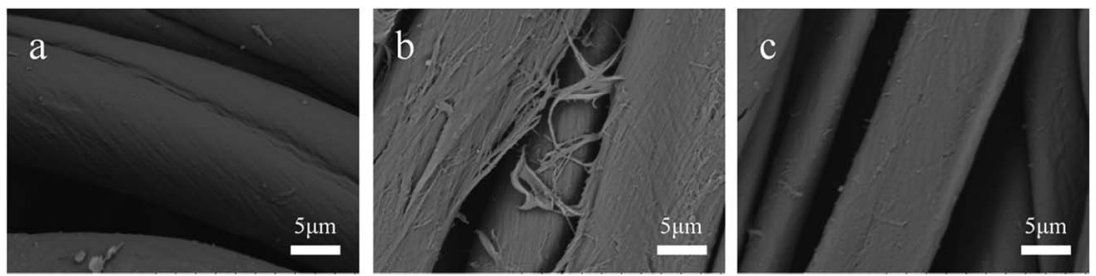

Fig. 3 SEM images of the cotton fibers: (a) untreated, (b) KH-580-pretreated, and (c) KH-580-GTAC-treated.

humidity until a constant weight was reached. Moreover, the test was carried out in a climate chamber at a specific temperature and relative humidity.

\section{Results and discussion}

The chemical composition of the fiber surface was investigated by EDS, and the spectra are shown in Fig. 1. As shown in Fig. 1(b), Si and S from 3-mercaptopropyltriethoxysilane were detected. The low content of Si and S may be due to the relatively low reaction rate of the modification agents on the cotton fibers. For the KH-580-GTAC-treated cotton, peaks attributed to $\mathrm{N}$ and $\mathrm{Cl}$ from GTAC were observed. The data provided sufficient evidence of a successful pretreatment and indicated that the reaction between thiol and epoxy groups by click chemistry had occurred.

Table 1 Number of colonies and antibacterial activity (according to AATCC 100-2004)

\begin{tabular}{|c|c|c|c|c|}
\hline Samples & $\begin{array}{l}\text { E. coli } \\
\text { (CFU) }\end{array}$ & $\begin{array}{l}\text { Antibacterial } \\
\text { activity }\end{array}$ & $\begin{array}{l}\text { S. aureus } \\
\text { (CFU) }\end{array}$ & $\begin{array}{l}\text { Antibacterial } \\
\text { activity }\end{array}$ \\
\hline Untreated & 180 & - & 123 & - \\
\hline KH-580-GTAC-treated & 1 & $99.44 \%$ & 5 & $95.93 \%$ \\
\hline $\begin{array}{l}\text { After } 30 \text { cycles of } \\
\text { washing }\end{array}$ & 11 & $93.89 \%$ & 14 & $88.62 \%$ \\
\hline
\end{tabular}

To confirm the thiol-epoxy reaction, the untreated and treated cotton samples were analyzed by FT-IR. Upon comparing IR spectra in Fig. 2(a) and (b), it can be observed that the cotton fabric after KH-580 modification contains thiol groups, evidenced by the peak at $2510 \mathrm{~cm}^{-1}$. As expected, the thiol peak disappeared after thiol groups reacted with the epoxy group of GTAC. Therefore, the IR results also confirmed the chemical bonding between GTAC and the cotton fabric samples.

The morphology of the untreated and treated fiber surfaces was examined by SEM, and the images are shown in Fig. 3. The original cotton fiber surface is relatively rough and uneven, as seen in Fig. 3(a), which had a deep ravine in the center and thin lines on both sides. However, the deep gully and clear lines on the surface of $\mathrm{KH}-580$ pretreated cotton gradually disappeared and the surface became fuzzy, as seen in Fig. 3(b), such that the surface is cross-linked into one layer, and the thiol group is attached. As can be seen from Fig. 3(c), the surface of the $\mathrm{KH}^{-}$ 580-GTAC-treated fiber becomes sparse and cross-linked, which may explain the antibacterial properties.

The antibacterial properties were measured according to the AATCC100-2004 standard. Table 1 shows the raw data. Fig. 4(a)(c) show the results for the antibacterial properties against $E$. coli and Fig. 4(d)-(f) are the results for the antibacterial properties against $S$. aureus of the untreated and KH-580-GTACtreated cotton fabric samples, and the treated cotton fabric samples after 30 cycles of washing. The number of colonies on the surface of the $\mathrm{KH}-580$-GTAC-treated cotton is significantly
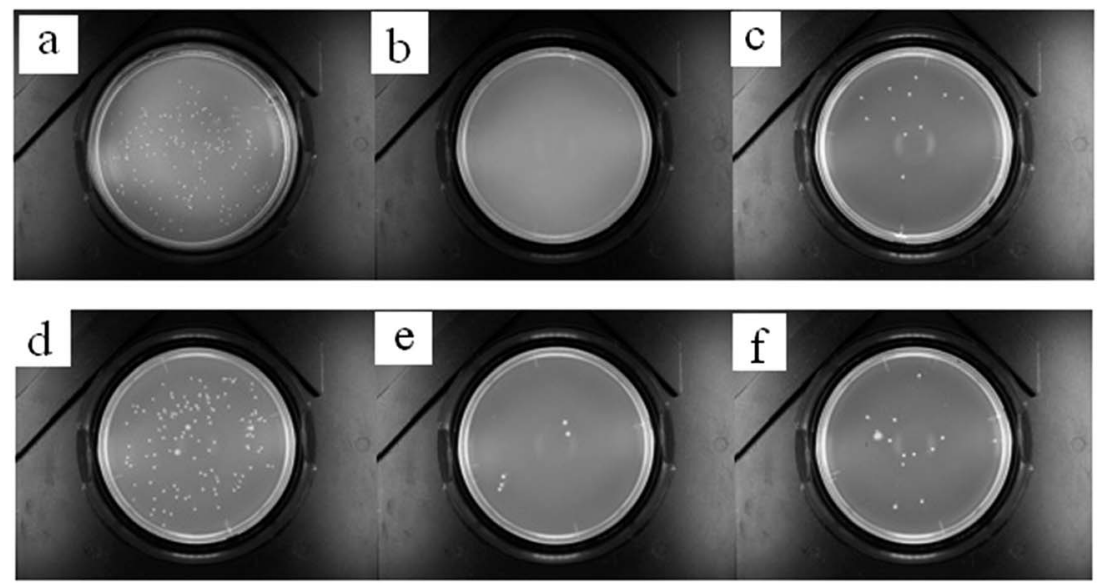

Fig. 4 Antibacterial activity of the cotton samples against E. coli: (a) untreated, (b) KH-580-GTAC-treated, and (c) KH-580-GTAC-treated cotton fabric after 30 cycles of standard washing. Against S. aureus: (d) untreated, (e) KH-580-GTAC-treated, and (f) KH-580-GTAC-treated cotton fabric after 30 cycles of standard washing. 

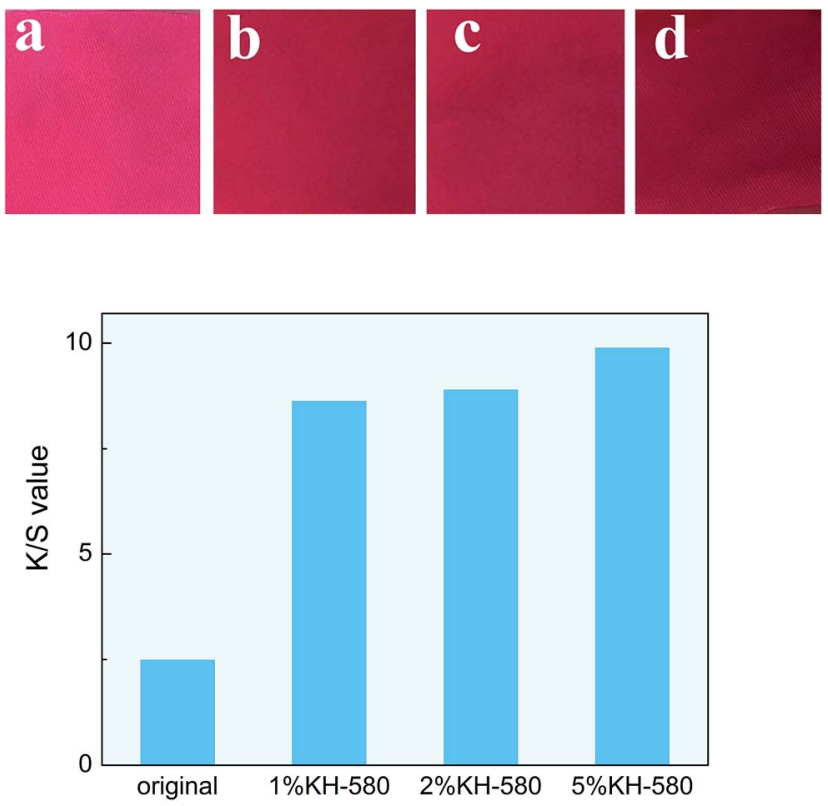

Fig. $5 \mathrm{~K} / \mathrm{S}$ values of different cotton samples: (a) untreated; (b) $1 \% \mathrm{KH}-$ 580 -treated; (c) $2 \% \mathrm{KH}-580$-treated; (d) $5 \% \mathrm{KH}-580$-treated.

lower. The $E$. coli antibacterial rate was about $99.44 \%$ and the $S$. aureus was about $95.93 \%$, which indicates obvious antibacterial properties. After 30 cycles of washing, the E. coli and S. aureus antibacterial rate respectively decreased to $93.89 \%$ and $88.62 \%$, indicating a high durability of the antibacterial performance.

Fig. 5 shows the maximum $K / S$ values of the untreated and treated cotton with a 1-5 wt $\% \mathrm{KH}-580$. Clearly, as the concentration of $\mathrm{KH}-580$ increased, the $K / S$ value gradually increased. Furthermore, the $5 \mathrm{wt} \% \mathrm{KH}-580$-treated cotton has the largest $K / S$ value, which indicates a larger amount of thiol groups and improved dyeing performance of the treated cotton fabric. After treatment, the samples contained more quaternary ammonium groups, which being positively charged attracted more negatively charged groups of the reactive dyes, thus improving the dyeing performance.

To analyze the mechanical properties of the treated cotton fabric, the fiber strength of different cotton fabrics was tested and the results are shown in Table 2. The strength of the original cotton yarn was $5.97 \mathrm{~N}$. A slight loss of the strength was observed after KH-580 pretreatment and after GTAC treatment. Five yarns per sample were measured and the test results were achieved by taking the average value. The results show that the treatment does not cause considerable damage to the fibers as the yarn strength seems to only slightly decrease.

Table 2 Yarn strength loss of the untreated and treated cotton samples

\begin{tabular}{lll}
\hline Samples & $\begin{array}{l}\text { Average yarn } \\
\text { strength }(\mathrm{N})\end{array}$ & $\begin{array}{l}\text { Strength retention } \\
(\%)\end{array}$ \\
\hline Untreated cotton & 5.97 & 100 \\
KH-580-treated cotton & 5.54 & 92.8 \\
KH-580-GTAC-treated cotton & 5.17 & 86.6
\end{tabular}

\section{Conclusions}

This study introduces an effective method based on thiol-epoxy click chemistry whereby durable antibacterial properties can be imparted to cotton fabrics. This treatment involves a mercaptomodification of the cotton fabric and the chemical bonding between GTAC and the substrate. EDS indicated that KH-580 was successfully grafted onto the cotton fabric, which was further verified by FTIR, and demonstrated the chemical bonding formed by quaternary ammonium salt and the substrate. The antibacterial efficiencies of the KH-580-GTACtreated cotton fabric and the fabric after 30 cycles of washing were $99.44 \%$ and $93.89 \%$, respectively, against E. coli. The antibacterial efficiencies of the KH-580-GTAC-treated cotton fabric and the fabric after 30 cycles of washing were $95.93 \%$ and $88.62 \%$, respectively, against $S$. aureus. Moreover, the $K / S$ values of the treated cotton fabrics were more than twice as large as those of the untreated cotton fabric, and the $5 \mathrm{wt} \% \mathrm{KH}-580$ treated cotton had the largest $K / S$ value, which indicated the improvement of the dyeing performance after treatment. Furthermore, the strength test results demonstrated that the treatment caused very little damage to the fibers. In view of these results, we can conclude that this method provides an effective way to modify cotton fabrics to achieve durable antibacterial properties.

\section{Acknowledgements}

This research was supported by the National Natural Science Foundation (no. 51403032, China).

\section{References}

1 M. Messaoud, E. Chadeau, P. Chaudouët, N. Oulahal and M. Langlet, J. Mater. Sci. Technol., 2013, 30, 19-29.

2 X. Fu, Y. Shen, X. Jiang, D. Huang and Y. Yan, Carbohydr. Polym., 2011, 85, 221-227.

3 C. E. Zhou and C. W. Kan, Appl. Surf. Sci., 2015, 328, 410-417.

4 M. Sundrarajan and A. Rukmani, Adv. Chem. Lett., 2013, 1, 40-43.

5 A. R. Shirvan, N. H. Nejad and A. Bashari, Fibers Polym., 2014, 15, 1908-1914.

6 C. J. Zhang, H. Yang, Y. Liu and P. Zhu, Adv. Mater. Res., 2011, 332-334, 77-80.

7 C. H. Kim, J. W. Choi, H. J. Chun and K. S. Choi, Polym. Bull., 1997, 38, 387-393.

8 L. Huang, X. Sun, Y. H. Xiao, Y. Dong, Z. C. Tong, X. D. Xing, F. Li, Z. G. Chai and J. H. Chen, J. Biomed. Mater. Res., Part B, 2012, 100, 1353-1358.

9 S. Chen, S. Chen, S. Jiang, M. Xiong, J. Luo, J. Tang and Z. Ge, ACS Appl. Mater. Interfaces, 2011, 3, 1154.

10 Y. H. Wang and X. U. Gao-Tian, Chem. Eng., 2004, 111, 12.

11 R. T. Carson, E. Larson, S. B. Levy, B. M. Marshall and A. E. Aiello, J. Antimicrob. Chemother., 2008, 62, 1160-1162.

12 M. B. Harney, R. R. Pant, P. A. Fulmer and J. H. Wynne, ACS Appl. Mater. Interfaces, 2009, 1, 39-41. 
13 P. Majumdar, E. Lee, N. Patel, S. J. Stafslien, J. Daniels and B. J. Chisholm, J. Coat. Technol., 2008, 5, 405-417.

14 I. Yudovin-Farber, N. Beyth, E. I. Weiss and A. J. Domb, J. Nanopart. Res., 2009, 12, 591-603.

15 Z. Shi, K. G. Neoh and E. T. Kang, Ind. Eng. Chem. Res., 2006, 46, 439-445.

16 J. Guo, Z. Xie, R. T. Tran, D. Xie, D. Jin, X. Bai and J. Yang, Adv. Mater., 2014, 26, 1906-1911.

17 S. N. Jampala, M. Sarmadi, E. B. Somers, A. C. Wong and F. S. Denes, Langmuir, 2008, 24, 8583-8591.

18 P. Thirumurugan, D. Matosiuk and K. Jozwiak, Chem. Rev., 2013, 113, 4905-4979.

19 J. E. Moses and A. D. Moorhouse, Chem. Soc. Rev., 2007, 38, 1249-1262.
20 W. Tian, Y. Hu, W. Wang and D. Yu, $R S C A d v ., 2015,5$, 91932-91936.

21 D. Yu, J. Y. Cai, J. S. Church and L. Wang, ACS Appl. Mater. Interfaces, 2014, 6, 1236-1242.

22 Q. Tu, C. Tian, T. Ma, L. Pang and J. Wang, Colloids Surf., B, 2016, 141, 196-205.

23 W. Tian, Y. Hu, W. Wang and D. Yu, $R S C A d v ., 2015,5$, 91932-91936.

24 Y. Hu, W. Wang, L. Xu and D. Yu, Mater. Lett., 2016, 178, 159-162.

25 W. Wang, W. Li, C. Gao, W. Tian, B. Sun and D. Yu, Appl. Surf. Sci., 2015, 342, 120-126. 